\title{
Astrobiology on the Dwarf Planet Ceres
}

\section{Chandra Wickramasinghe $\mathbf{N}^{1,2,3}$}

${ }^{1}$ Buckingham Centre for Astrobiology (BCAB), Buckingham University, UK

${ }^{2}$ Institute for the Study of Panspermia and Astroeconomics, Gifu, Japan

${ }^{3}$ University of Peradeniya, Peradeniya, Sri Lanka

\section{Abstract \\ With NASA's Dawn probe going into orbit around Ceres, the largest object in the asteroid belt will become a focus of astrobiological interest.}

Keywords: Ceres; Dwarf planets; Microbiology; Panspermia; Fred Hoyle

\section{Editorial}

In a remarkable instance of serendipity the present centenary year of Fred Hoyle (1915-2015) has been marked by several landmark developments in astrobiology. Fred Hoyle was the iconic astrophysicist of the $20^{\text {th }}$ century who arguably pioneered modern astrobiology, and in particular ideas relating to panspermia [1]. Several recent developments in astrobiology, all pointing to the vindication of panspermia, have been discussed elsewhere in this journal [2-4].

The dwarf planet Ceres (perihelion 2.56AU; aphelion 2.98AU), which is located in the asteroid belt between the orbits of Mars and Jupiter, may not have ranked high as a candidate for astrobiological exploration. The changed, however, after emissions of water vapour were discovered by the Hershel Space Telescope [5].

Ceres is a roughly spherical planetary body comprised of a silicate/iron core surrounded by an extensive ice mantle of radius approximately $500 \mathrm{~km} \mathrm{[6].} \mathrm{An} \mathrm{average} \mathrm{surface} \mathrm{temperature} \mathrm{of} \mathrm{minus}$ 100 degrees $\mathrm{C}$ and low gravity will not permit the persistence of water vapour in its atmosphere; nor could there be an appreciable rate of $\mathrm{H}_{2} \mathrm{O}$ sublimation from a surface at such a low temperature. Observations with the Hershel telescope revealing plumes of water vapor emerging at a prodigious rate of $6 \mathrm{~kg}$ per second from localized regions of Ceres thus came as a surprise [5]. These observations are consistent, however, with the build-up of pockets of high pressure gas in subsurface lakes that are occasionally vented though cracks and fissures in weak spots of the overlying ice.

For an object of the size of Ceres, the heat released by the decay of radioactive nuclides $\left({ }^{26} \mathrm{Al},{ }^{60} \mathrm{Fe},{ }^{238} \mathrm{U}\right)$ will serve to melt large volumes of interior ice. It is a fair bet that these interior lakes would be replete with organic molecules serving as habitats for hetero autotrophic microorganisms. Due to the very low thermal conductivity of overlying ice (and organics) subsurface lakes would remain in a warm liquid state for timescales that are well in excess of the age of the solar system [7]. A possible explanation for the water vapour emission from Ceres is similar to that described earlier to explain similar processes in comets [8]. Biological activity in warm subsurface lakes leads to the build-up of high enough pressures of gaseous metabolites to occasionally rupture overlying layers of ice.

The recently reported discovery of exceptionally bright spots in the basin of a $92 \mathrm{~km}$-wide crater may provide startling evidence of this process (http://www.jpl.nasa.gov/news/news.php?feature=4491). The image released by NASA is reproduced in (Figure 1). It could be argued that pressure-induced fissures in the crater floor have recently refrozen

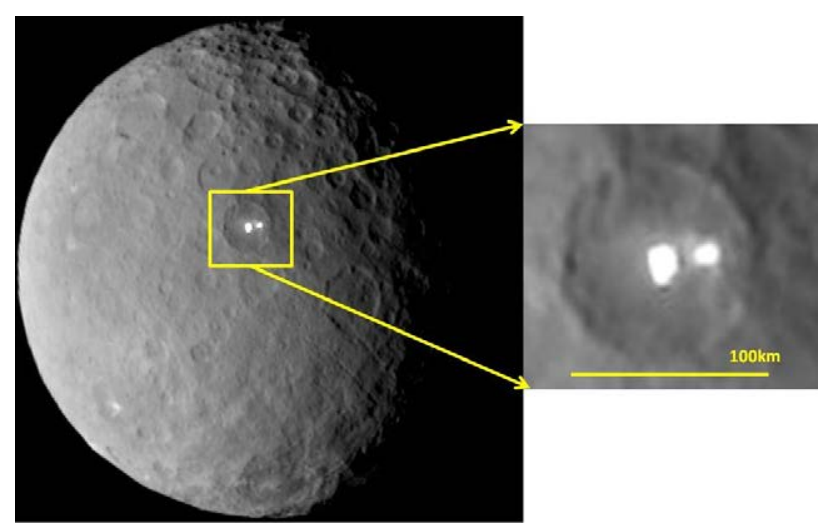

Figure 1: Adapted from image of Ceres taken by NASA's Dawn spacecraft on Feb. 19, 2015 at a distance of nearly $46,000 \mathrm{~km}$. Note that the brightest spot on Ceres (see inset) has a dimmer companion, which apparently lies in the same crater basin. Courtesy: NASA/JPL-Caltech/UCLA/MPS/DLR/IDA.

to produce smooth icy regions of diameter $<<10 \mathrm{~km}$, the resolution limit claimed for this image. Smooth, clean ice and snow would possess a very high albedo and contrast sharply with a dust covered dirty icesiliceous surface. This could explain the bright spots. The spacecraft Dawn, that took the image in (Figure 1), is now securely in orbit around Ceres. We may hope that the mystery of the bright spots will be solved in the near future, and that the astrobiology of this dwarf planet will be established on a firm footing.

Watch this space!

\section{References}

1. Wickramasinghe $C$ (2002) The beginnings of astrobiology. Int. J. Astrobiol 1 77-78.

2. Wickramasinghe NC, Tokoro $\mathrm{G}$ (2014) Life as a cosmic phenomenon 1; The socio-economic control of a scientific paradigm. Journal of Astrobiology and Outreach 2: 113

3. Tokoro G, Wickramasinghe NC (2014) Life as a cosmic phenomenon 2; The

*Corresponding author: Wickramasinghe NC, Buckingham Centre for Astrobiology (BCAB), Buckingham University, UK, Tel: +44-777-838-9243; E-mail: ncwick@gmail.com

Received March 07, 2015; Accepted March 09, 2015; Published March 11, 2015

Citation: Chandra Wickramasinghe N (2015) Astrobiology on the Dwarf Planet Ceres. Astrobiol Outreach 3: e108. doi:10.4172/2332-2519.1000e108

Copyright: @ 2015 Chandra Wickramasinghe N. This is an open-access article distributed under the terms of the Creative Commons Attribution License, which permits unrestricted use, distribution, and reproduction in any medium, provided the original author and source are credited. 
panspermic trajectory of Homo sapiens. Journal of Astrobiology and Outreach 2: 115

4. Wickramasinghe NC, Wainwright M, SmithWE, Tokoro G, Al-Mufti S (2015) Rosetta studies of Comet 67P/Churyumov-Gerasimenko: Prospects for establishing cometary biology. Journal of Astrobiology and Outreach 3: 126.

5. Küppers M, Laurence OR, Morvan DB, Zakharov V, Lee S, et al. (2014) Localised sources of water vapour on dwarf planet (1) Ceres. M. Nature 505: $525-527$.
6. Castillo-Rogez JC, McCord TB (2010) Ceres' evolution and present state constrained by shape data. Icarus 205: 443-459.

7. Wickramasinghe J, Wickramasinghe NC, Napier WM (2013) Comets and the origins of life, World Scientific, Singapore.

8. Wickramasinghe NC, Hoyle F, Lloyd D (1996) Eruptions of comet Hale-Bopp at 6.5 AU. Astrophys. Sp. Sci 240: 161-165. 